\title{
LOS JÓVENES Y LA GLOBALIZACIÓN. UN ENFOQUE DESDE EL FINANCIAMIENTO A LA SEGURIDAD SOCIAL*
}

\author{
YOUNGSTERS AND GLOBALIZATION. AN APPROACH \\ FROM SOCIAL SECURITY FUNDING
}

LES JEUNES ET LA MONDIALISATION. UN APPROCHE
À PARTIR DU FINANCEMENT DE LA SÉCURITÉ SOCIALE

Juan Manuel GÓMEZ RoDRÍGUEZ**

\begin{abstract}
RESUMEN: Este artículo explora el fenómeno de la globalización y su impacto en la juventud desde la perspectiva del financiamiento a la seguridad social. Se analiza, en primer lugar, la exclusión social en general y la retirada del derecho social en las políticas públicas para los jóvenes. En segundo término, después de analizar sus consecuencias, se abordan los diferentes enfoques de la exclusión social. Se concluye que las políticas orientadas por estímulos fiscales que promuevan la seguridad social en el empleo juvenil se vuelven un elemento esencial de su financiamiento y contribuyen a estimular un mejor aprovechamiento de los jóvenes respecto a las oportunidades que brinda la globalización.
\end{abstract}

Palabras clave: Jóvenes, globalización, exclusión social, sistema educativo, delincuencia.

ABSTRACT: This article explores the phenomenon of globalization and its impact on youth from the perspective of social security funding. Firstly, it examines social exclusion in general and the removal of Labour Law in public policies for youth. Secondly, after analyzing its consequences, different approaches to social exclusion are addressed. We conclude that tax-incentive oriented policies that promote social security on youth employment become an essential element to its funding and

\footnotetext{
* Recibido el 23 de marzo de 2012 y aceptado para su publicación el 10 de abril de 2012.

** Doctor en derecho comunitario europeo por la Universidad de Castilla-La Mancha, España; profesor investigador de tiempo completo en la Facultad de Derecho y Ciencias Sociales de la Universidad Autónoma del Estado de Morelos, México (jm2oct@yahoo.com).
} 
help encourage a better use of the youth regarding the opportunities offered by globalization.

Key Words: Youth, globalization, social exclusion, education system, criminality.

RÉSUMÉ: L'article explore le phénomène de la mondialisation et son impact sur les jeunes dans la perspective du financement de la sécurité sociale. Il examine d'abord l'exclusion sociale générale et le retrait du droit du travail dans les politiques publiques pour les jeunes. En suite, après avoir analysé ses conséquences, sont traitées les différentes approches sur l'exclusion sociale. On conclut que les politiques orientées par des stimulations fiscales qui promeuvent la sécurité sociale pour l'emploi juvénile se retournent un élément essentiel de son financement et contribuent à stimuler un meilleur profit des jeunes par rapport aux opportunités que la globalisation offre.

Mots-clés: Jeunesse, mondialisation, exclusion sociale, système éducatif, criminalité.

SUMARIO: I. Introducción. II. La exclusión social. III. Maffesoli y El tiempo de las tribus. IV. La retirada del derecho social en las políticas públicas, una explicación a la exclusión social con motivo de la globalización. V. La exclusión social en el sistema educativo. VI. El deterioro de las condiciones laborales como causa de la delincuencia en los jóvenes. VII. La criminalización y su influencia en la exclusión social de la juventud. VIII. Los jóvenes y la seguridad social. Un enfoque respecto al financiamiento. IX. Conclusiones. X. Bibliografía.

\section{INTRODUCCIÓN}

0 i realizamos un ejercicio empírico de observación podríamos afirmar que los comportamientos y actitudes de los jóvenes de nuestro entorno actual son fruto de la globalización, los medios de comunicación, la pérdida de valores morales, religiosos y familiares, así como el deterioro de la esperanza en las instituciones públicas para resolver los problemas de pobreza, exclusión de oportunidades y desigualdad que la misma dinámica global han generado. También podríamos agregar que los problemas económicos son un elemento constante de la juventud, cuyas causas implican analizarla desde 
la perspectiva de las características de clase, raza, religión y pertenencia o no a un grupo social determinado. En ese sentido, la juventud es más que una palabra. ${ }^{1}$

¿Cuáles podrían ser concepciones de la juventud? A los jóvenes se les ha visto desde dos perspectivas: como sujetos de cambio y como problema social. La primera mirada concibe a la juventud desde la transgresión social y la rebeldía; encargándose de dirigir las transformaciones sociales y la modernización. En palabras de Rodríguez, hay una contribución sustancial de la juventud a la construcción y renovación de la sociedad. ${ }^{2}$ Desde las manifestaciones colectivas como los movimientos estudiantiles o las expresiones estéticas en la vestimenta, la moda o la música. ${ }^{3}$

Por otro lado, se visualiza a la juventud como un problema social, donde existen dificultades que conllevan a la delincuencia, la anarquía, la drogadicción, el fracaso escolar, etcétera. ${ }^{4} \mathrm{Si}$ bien esta perspectiva estudia grupos específicamente marginados, en esencia se contempla el fenómeno juvenil como un estado de suspensión entre la niñez y la vida adulta.

Sin embargo, una aproximación más analítica vislumbra a la juventud como una manera de ser en sí, válida sin sujetarla a criterios de maduración o integración, más bien como la esencia mutable socialmente construida. Culturalmente, única e irrepetible en el lugar y en el momento histórico.

Así, la concepción de la juventud deja de ser un mero acercamiento a una población intermedia entre la niñez y la vida adulta, para comprender las causas que hacen que los jóvenes se identifiquen como tales en determinado tiempo y lugar, con características propias y necesidades específicas. Este trabajo tiene como objetivo analizar el fenómeno de la globalización y su impacto en la juventud desde la perspectiva del financiamiento a la seguridad

1 Bourdieu, Pierre, "La juventud no es más que una palabra”, Sociología y Cultura, México, CNCA-Grijalbo, 1990.

2 Rodríguez, Ennesto, "Políticas públicas de la juventud en América Latina: experiencias", Pensamiento Iberoamericano, España, AECID y Fundación Carolina, núm. 3, 2008, pp. 273 y ss.

3 Medina Carrasco, Gabriel, Aproximaciones a la diversidad juvenil, México, El Colegio de México, 2000.

4 Véase Hopenhayn, Martin, "Inclusión social y exclusión social en la juventud latinoamericana”, Pensamiento Iberoamericano, España, AECID y Fundación Carolina, núm. 3, 2008, pp. 49-72, y Reguillo, Rossana, "Las múltiples fronteras de la violencia: jóvenes latinoamericanos entre la precarización y el desencanto", Pensamiento iberoamericano, España, AECID y Fundación Carolina, núm. 3, 2008, pp. 273-294. 
social. Nuestra premisa es que la globalización, si bien puede ser considerada como una palanca al desarrollo, también se constituye como una herramienta que promueve la exclusión social en los jóvenes, la falta de oportunidades, el desempleo y, como consecuencia, la carencia de seguridad social.

$\mathrm{Al}$ respecto, consideramos que la respuesta puntual a la problemática que presenta la juventud en nuestros días no sólo debe considerar la escasez de recursos y la pobreza, sino factores sociales como la exclusión social y sus diferentes enfoques, que se traducen en la carencia de políticas públicas, en particular la seguridad social enfocada a los jóvenes, que impiden, o bien obstaculizan, su inserción apropiada en la sociedad global que vivimos.

Recuperar políticas públicas que incorporen la protección y la seguridad social para los jóvenes se convierte por tanto en un elemento fundamental, de cara a conseguir una mejor capitalización de los diferentes sistemas de seguridad social en nuestro país y contribuir a una mejora sustancial en la calidad de vida de la sociedad mexicana, mediante el aprovechamiento puntual del bono demográfico que representa su juventud.

\section{LA EXCLUSIÓN SOCIAL}

El análisis de la juventud como categoría social presenta dos posibilidades metodológicas para su estudio: una de ellas es mediante los estudios culturales, y la otra es el enfoque hacia la diversidad juvenil. Para Foucault, los procesos sociales fundan las categorías analíticas a través de mecanismos de poder que legitiman y excluyen determinadas prácticas mediante una construcción socio-histórica justificada por la ciencia, la medicina, la religión y el derecho. De esta forma, hablamos de niños hiperactivos, atendidos por la medicina y la pedagogía; transgresores sociales, reprendidos por el derecho, y pecadores, condenados por la religión. ${ }^{5}$

Nosotros podemos agregar que esa construcción adicionalmente también está justificada por la economía, la política y la sociedad misma, y de esta forma hablamos también de jóvenes excluidos socialmente. De acuerdo con Giddens, ${ }^{6}$ la exclusión social alude a cómo los individuos pueden verse

5 Foucault, Michel, Historia de la sexualidad. 1. La voluntad de saber, México, Siglo XXI Editores, 1977.

6 Giddens, Anthony, Sociología, Madrid, Alianza, 2001, p. 415. 
apartados de una completa participación en el conjunto de la sociedad. El concepto se centra en una amplia gama de factores que impiden a individuos y grupos tener oportunidades con las que cuenta la mayoría de la población.

La exclusión puede abordarse, de acuerdo con este autor británico, desde tres diferentes puntos de vista:

1) La exclusión económica. Implica que los individuos y las comunidades pueden sentirse excluidos de la economía, tanto desde una perspectiva productiva como desde la del consumo. Del lado de la producción, el empleo y la participación en el mercado de trabajo son capitales para la inclusión. En comunidades en las que se registra un alto grado de privación material, como consecuencia de un desempleo generalizado, hay menos personas trabajando en jornada completa, y las redes informales que pueden ayudar a los parados a entrar en el mercado laboral son escasas.

La exclusión puede estar relacionada con las pautas de consumo, es decir, con lo que la gente compra y utiliza en su vida cotidiana; esto puede ejemplificarse en el acceso a la comunicación, como es el caso de la falta de teléfono o acceso a Internet, la falta de acceso al financiamiento bancario, a través de una cuenta corriente y, como consecuencia, a crédito para consumo, así como la falta de una vivienda que impide un lugar de residencia permanente y de desarrollo de la personalidad.

2) La exclusión política. La participación política activa puede no estar al alcance de los socialmente excluidos, que quizá carezcan de los recursos, de la información y de las oportunidades que se necesitan para implicarse en ese proceso. Para participar en grupos de presión, manifestaciones o mítines políticos, se precisa un grado de movilidad, un tiempo y un acceso a la información de los que pueden carecer las personas excluidas.

3) La exclusión social. La exclusión social también puede manifestarse en el ámbito social y en el de la vida comunitaria. Las áreas que sufren un alto grado de exclusión social pueden contar con pocos servicios comunitarios, como parques, instalaciones deportivas, centros culturales y teatros. La exclusión social también puede significar que las redes sociales son limitadas o débiles, lo cual produce aislamiento y un contacto mínimo con los demás. 
$\mathrm{Al}$ abordar el fenómeno de la exclusión social debemos ser conscientes, por una parte, de la interacción entre la capacidad de acción y la responsabilidad del ser humano y, por la otra, del papel que tienen las fuerzas sociales a la hora de conformar las circunstancias de las personas.

Ahora bien, la globalización plantea la necesidad de considerar un uso de los recursos humanos y materiales cada vez más eficiente. Esta perspectiva implica que los jóvenes sean cada vez más objeto de estudio por parte de los organismos internacionales. ${ }^{7}$

Un elemento de análisis que puede explicar este fenómeno radica en el hecho de que los jóvenes son vistos como actores estratégicos del desarrollo en una sociedad del conocimiento. Sin embargo, los diferentes programas utilizados desde el contexto institucional para el tratamiento de los jóvenes, reflejan que las políticas públicas destinadas a la juventud presentan fragmentación, desarticulación y duplicación de esfuerzos.

Una muestra clara de ello la constituye una inversión dispersa y fragmentada en programas que no guardan ninguna conexión o relación entre sí, la ausencia de prioridades estratégicas en los ámbitos de la educación y la salud para los jóvenes, pues los avances en estos ámbitos se concentran en la protección infantil y descuidan el acceso a la educación de calidad y de salud a jóvenes sin acceso a ellos o marginados, así como la escasa evaluación del uso de los recursos invertidos, lo que resulta en la falta de rendición de cuentas de los programas y políticas destinados a la juventud.

\section{MAFFESOLI Y EL TIEMPO DE LAS TRIBUS}

Para analizar el impacto que la globalización tiene en los jóvenes es pertinente considerar los profundos cambios sociales que plantea la globalización en la organización social. Hay quienes observan una retirada del individualismo hacia formas de organización colectivas en las que se puedan compartir e identificar necesidades y preferencias comunes.

7 Véase el Informe del Banco Mundial, El potencial de la juventud: políticas para jóvenes en situación de riesgo en América Latina y el Caribe, Washington, 2007. 
"Encontrar las palabras para expresar nuestro tiempo" es el objetivo de Mafessoli en su obra El tiempo de las tribus. ${ }^{8}$ En ella propone la metáfora de la "tribu" para dar cuenta de la metamorfosis del lazo social. La masificación característica de las ciudades, y en particular en las megalópolis, sería la arena ideal de surgimiento de esos microgrupos a los que Mafessoli llama "tribus urbanas". La causa del tribalismo, según este autor, estaría en las sociedades modernas, racionalizadas a ultranza, aseptizadas por excelencia, que se aplican a anular todo riesgo posible. La intuición como potencia societal, como afirmación de lo colectivo, sería la clave de este neo-tribalismo contemporáneo. Después de la dominación del "principio de logos", el de una razón mecánica y predecible, el de una razón instrumental y estrictamente utilitaria, asistimos al retorno del "principio del eros".

En este sentido, Mafessoli refiere que antes de ser político, económico o social, el tribalismo es un fenómeno cultural. Verdadera revolución espiritual. Revolución de los sentimientos que pone en relieve la alegría de la vida primitiva, alejada de los valores universalistas y racionalistas de la sociedad moderna. El progreso lineal y garantizado, causa y efecto de un evidente bienestar social, está siendo sustituido por una especie de "regresión", característica del tiempo de las tribus. Señala que "entrar (ingressa) sin progresar (progressa)" es lo que parece estar en juego en las sociedades contemporáneas. En el caso de los jóvenes, no los mueve una meta por alcanzar un proyecto económico, político o social por realizar. Prefieren "entrar en" el placer de estar juntos; "entrar en" la intensidad del momento; "entrar en" el goce del mundo tal cual es.

Esta actitud podría estar en el origen del por qué surgen las tribus urbanas. Para Maffesoli, las sociedades actuales carecen de "identidad"; ya no es a partir del individuo, poderoso y solitario, fundamento del contrato social, de la ciudadanía deseada o de la democracia representativa defendida como tal, que se constituye la vida en sociedad. Ésta es, ante todo, condicional, fusional, gregaria.

La explicación de la vida cotidiana por el individuo y el individualismo teórico que le sirve de fundamento ya no funcionan, lo que produce un sentimiento de desorientación en los jóvenes que les arrastra a entrar en este tipo de grupos. Se ha efectuado una saturación. Maffesoli argumenta que se trata

8 Maffesoli, Michel, El tiempo de las tribus. El ocaso del individualismo en las sociedades posmodernas, México, Siglo XXI Editores, 2004, pp. 26 y ss. 
del deslizamiento del individuo de identidad estable, ejerciendo su función dentro de los conjuntos contractuales, a la persona que desempeña papeles dentro de tribus afectuales.

Para Maffesoli, las instituciones ya no dotan de identidad a los individuos, lo que conlleva que los jóvenes que están desorientados, confusos, perdidos, disconformes, se agrupen en tribus urbanas. En las tribus urbanas los jóvenes encuentran una vía para establecer vínculos con los demás y obtener una identidad como grupo, así como una auto-afirmación. Las tribus urbanas serían una respuesta de los jóvenes a la falta de identidad, el desencanto social, la marginación o la exclusión social.

Un enfoque diferente es plantear el problema desde la perspectiva del acceso restringido a la participación ciudadana que enfrentan los jóvenes. La falta de espacios efectivos para la deliberación y la definición de roles y funciones, así como de responsabilidades, genera que los jóvenes no sean considerados en la formación de las políticas públicas que les atañen.

\title{
IV. LA RETIRADA DEL DERECHO SOCIAL EN LAS POLÍTICAS PÚBLICAS, UNA EXPLICACIÓN A LA EXCLUSIÓN SOCIAL CON MOTIVO
}

\author{
DE LA GLOBALIZACIÓN
}

La transición de la juventud a la edad adulta plantea muchos desafíos. Muchos jóvenes luchan por integrarse en la sociedad y se ven excluidos de ella de diversas formas. El primer problema tiene que ver con la transformación del mercado de trabajo. En la actualidad, el mercado laboral para los jóvenes es menos seguro que antes, lo cual hace que la transición desde el hogar paterno hasta la vida adulta independiente sea menos directa.

A muchos jóvenes les cuesta encontrar trabajo; los empleos no cualificados están dando lugar a puestos para los que se requieren ciertas habilidades o conocimientos de nuevas tecnologías. Los cambios en las prestaciones sociales también han afectado a las pautas de exclusión entre unos jóvenes que ven reducidos sus derechos sociales en relación con el empleo, la educación y la vivienda.

Otro motivo de preocupación es el hecho de que el sistema educativo está excluyendo a un número creciente de jóvenes, tanto formal como informalmente. Las políticas actuales que fomentan la selección dentro de las escuelas, 
y entre ellas, tienden a perjudicar a los jóvenes, que ya de por sí están situados en los márgenes de la sociedad. ${ }^{9}$

Un problema vinculado a la marginación en el mercado laboral y en el sistema educativo es la exclusión de los jóvenes a la vivienda, que constituye en la actualidad una de las principales manifestaciones de exclusión social, que se manifiesta en indigencia y en la falta de espacios apropiados para el desarrollo de los jóvenes. El sector inmobiliario no está bien equipado para afrontar las pautas cambiantes de movilidad juvenil. Los jóvenes disponen de pocos recursos y no pueden acceder a viviendas adecuadas para ellos. Quienes carecen de residencia permanente pueden verse completamente apartados de muchas de las actividades cotidianas que otros dan por hechas, como ir a trabajar, tener una cuenta corriente, recibir amigos, o incluso recibir correspondencia.

En la actualidad podría señalarse que la falta de vivienda incide también en la falta de acceso a nuevas tecnologías como el internet y las redes sociales, considerados claves para el desarrollo social en los actuales tiempos de globalización que vivimos.

En ese sentido, es posible observar que una deficiente regulación laboral que inhibe la inserción laboral de los jóvenes en condiciones adecuadas, la falta de un acceso adecuado para la formación y capacitación laboral de los jóvenes, por ejemplo en el uso de nuevas tecnologías y en el desarrollo de nuevas habilidades y destrezas orientadas a cubrir las necesidades que el mercado laboral demanda, así como la exclusión de los jóvenes a la vivienda, pueden explicarse en el retiro o ausencia del derecho social de las políticas sociales, representado en la restricción de los derechos laborales, de educación, de salud y de vivienda, iniciado precisamente con las políticas de apertura hacia el mercado, así como el retroceso de las prestaciones sociales, no sólo respecto a los trabajadores, sino principalmente respecto a grupos vulnerables como los jóvenes.

\section{LA EXCLUSIÓN SOCIAL EN EL SISTEMA EDUCATIVO}

El sistema educativo es como una gran carrera de obstáculos. Existe un alto número de abandonos prematuros y de participantes descalificados por

9 France, Alan y Wiles, Paul, "Dangerous Futures: Social Exclusion and Rough Work in Late Modernity", en Jones Finer, Catherine y Nellis, Mike (eds.), Crime and Social Exclusion, Oxford, Blackwell, 1998. 
no haber cubierto la distancia mínima en el plazo establecido. La idea de que la formación escolar es, por excelencia, el espacio natural de igualdad de oportunidades debe matizarse atendiendo no sólo al esfuerzo y a la capacidad personal como elementos relevantes. Los jóvenes no parten de la línea de salida en condiciones idénticas y con competencias similares. En el caso de España, por ejemplo, la escolarización obligatoria hasta los 16 años se ha revelado como uno de los sistemas educativos más equitativos de la OCDE, de acuerdo con el informe PISA, que evalúa el nivel de conocimientos de los jóvenes de 15 años de 55 países del mundo. El Informe señala que si se eliminan los condicionantes socioeconómicos y culturales de los alumnos, las escuelas españolas públicas, privadas y concertadas dan unos resultados muy similares entre sí. Los hijos de los trabajadores no cualificados tienen 4.5 veces menos de probabilidades de acceder al ámbito universitario que los hijos de profesionales de alto nivel. Sólo un tercio de los de familias obreras o de asalariados del campo cursará el bachillerato, y de ellos únicamente la mitad llegará a la universidad. Los hijos de padres que no tienen estudios tienen 20 veces más de posibilidades de incurrir en el fracaso escolar que el hijo de padres universitarios; exactamente el $40 \%$ frente al 2\%, según el estudio recientemente publicado por José Saturnino Martínez, profesor de Sociología de la Universidad de la Laguna. ${ }^{10}$

El sistema educativo se convierte, por tanto, en una maquinaria de la reproducción de las desigualdades socioeconómicas, sin perjuicio de que en el caso de alumnos, particularmente brillantes y trabajadores, deje márgenes de maniobra para "la movilidad de clase" y haya acompañado la irrupción de las mujeres, cuyo rendimiento es muy superior. No obstante, gracias a las becas siguen dándose ejemplos de alumnos de familias de rentas muy bajas que acaban hasta dos carreras universitarias. Sin embargo, no dejan de ser una notable excepción en un modelo en el que el capital cultural y económico condiciona fuertemente el rendimiento escolar y el estatus social. Como han puesto de relieve los economistas Jorge Calero y Josep-Oriol Escardíbul, la educación determina cada vez más la posición laboral y las trayectorias vitales de las personas. ${ }^{11}$

10 Barbería, José Luis, "La clase perdedora”, en El País, 7 de abril de 2009.

11 Idem. 
Las estadísticas hablan de un problema colectivo que además de socavar la equidad y la justicia comprometen el futuro de cualquier país, arrojando al mercado de trabajo a masas de jóvenes poco cualificados para afrontar "la sociedad del conocimiento". De hecho, las diferencias de rendimiento escolar registradas en el informe PISA se explican básicamente por el nivel social tanto de los padres como de los centros. Los investigadores han llegado a la conclusión de que la variabilidad observada entre centros educativos en las pruebas de lectura está asociada en un 50\% a las características del estudiante, muy particularmente al estatus socioeconómico de su familia y también al sexo, la edad y la condición o no de inmigrante. Las características del centro influirían en los resultados en un $16 \%$, mientras que la naturaleza competitiva o cooperativa de los métodos didácticos, los medios materiales y el tipo de gestión no superarían el 6\%.

La mayoría de los expertos opina que el nivel cultural de los padres pesa más que sus recursos económicos. Remover las desigualdades sociales requiere que la educación sea lo más independiente posible de las condiciones socioeconómicas de los alumnos.

En el caso de México, la situación no podría ser peor. Pese al esfuerzo en destinar cada vez mayores recursos a la educación básica, esto no se ha traducido en una educación de mayor calidad para afrontar la economía del conocimiento que se necesita para el siglo XXI. Datos del Programa para la Evaluación Internacional de los Estudiantes (PISA), realizado por la Organización de Cooperación y Desarrollo Económico (OCDE), revelan que México ocupa el último lugar en las evaluaciones respecto al desarrollo de las habilidades de lectura, matemáticas y ciencia. ${ }^{12}$

\section{EL DETERIORO DE LAS CONDICIONES LABORALES COMO CAUSA DE LA DELINCUENCIA EN LOS JÓVENES}

Algunos autores han señalado que existe un fuerte vínculo entre la delincuencia y el deterioro de las condiciones laborales y la exclusión social. Indican que en las sociedades modernas se abandonan los objetivos incluyentes y

12 Véase http:// mmm.oecd.org/document/61/0,3746,en_32252351_32235731_46567613_1_1_ 1_1,00.htm. 
se opta por arreglos que aceptan o incluso fomentan la exclusión de algunos ciudadanos. ${ }^{13}$

En México, en los últimos años es posible observar las consecuencias de una brutal apertura de la economía hacia el libre mercado; el incremento de la pobreza y la indigencia, el notable incremento de los delitos violentos y un floreciente consumo de drogas entre los jóvenes. Hay varias posibles causas que pueden influir en este fenómeno; la falta de formación en los hogares es uno de ellos. Debido a las carencias económicas, los padres requieren trabajar de tiempo completo y en actividades diversas, y es más frecuente que los jóvenes se eduquen solos, sin la orientación y el apoyo que necesitan de la población adulta.

Los adultos de muchas familias pobres se ven obligados a aceptar múltiples trabajos para sobrevivir, lo que provoca tensión constante, ansiedad y ausencia de casa. Otro elemento a considerar es la reducción de las oportunidades laborales con las que ganarse la vida. Para Currie, es posible considerar que en sus comunidades los jóvenes sufran la pérdida de los medios para ganarse una vida estable, que los obligue a emigrar a otras ciudades; la privación económica y la fragmentación de la comunidad presionan sobre la vida familiar. Esta situación puede producir una profunda sensación de privación relativa y una disposición a orientarse hacia formas ilegítimas de mantener la forma de vida que se desea. ${ }^{14}$

Los medios de comunicación masiva, como la televisión o el Internet, fomentan el consumo, y presionan a los jóvenes para alcanzar la posición económica y la capacidad de consumo que se fomentan dentro de la sociedad; no obstante, los jóvenes, en la mayor parte de los casos, no tienen la capacidad económica ni el acceso a las formas legítimas para alcanzarla, es decir, las oportunidades necesarias para su inserción apropiada al mercado laboral están vinculadas en proporción directa con su grado de estudios y su experiencia laboral previa. Esta situación puede llevar a los jóvenes a buscar en la delincuencia, el instrumento para hacerlo y soslayar a la organización política

13 Young, Jock, "Breaking Windows: Situating the New Criminology", en Walton, Paul y Young, Jock (eds.), The Exclusive Society: Social Exclusion, Crime and Difference in Late Modernity, Londres, Sage, 1998.

14 Currie, Elliot, "Crime and Market Society: Lessons from the United States", en Walton, Paul y Young, Jock (eds.), The New Criminology Revisited, Londres, Macmillan, 1998. 
y sus instituciones como instrumentos efectivos en el diseño de políticas públicas que incidan de manera efectiva en un acceso a mejores oportunidades de desarrollo para los jóvenes.

\section{LA CRIMINALIZACIÓN Y SU INFLUENCIA EN LA EXCLUSIÓN SOCIAL DE LA JUVENTUD}

La cobertura que hacen los medios de comunicación de las crecientes tasas de criminalidad se ocupa principalmente de la decadencia moral que reina entre los jóvenes y subraya problemas como el vandalismo, el absentismo escolar y el consumo de drogas, como ejemplos del aumento de la permisividad social. Si bien es cierto que las estadísticas revelan la alta incidencia de la criminalidad en los jóvenes, los presupuestos entre juventud y delincuencia deben abordarse de manera cuidadosa.

Para Muncie, los pánicos morales que tienen por objeto la delincuencia juvenil no reflejan fielmente la realidad social. Un delito aislado en el que se hayan visto implicados jóvenes puede constituirse en el motivo o pretexto necesario para una enérgica respuesta por parte de la ley y el orden.

La misma cautela ha de mostrarse con la idea tan extendida de que la mayoría de los delitos tiene que ver con drogas. Hay estudios recientes que revelan que el consumo de drogas y de alcohol entre los jóvenes está en un nivel relativamente normal. Una encuesta realizada a siete mil adolescentes de entre quince y dieciséis años en el Reino Unido puso de manifiesto que más del 94\% tomaba bebidas alcohólicas, cerca de un tercio había fumado un cigarro en los treinta días anteriores y el $42 \%$ había probado drogas ilegales al menos una vez. ${ }^{15}$

En algunos casos, la estigmatización anticipada juega un papel muy importante. Es una especie de profecía que se cumple a sí misma. Tradicionalmente se ha caracterizado al delincuente juvenil como varón, joven y pobre. Sin embargo, hay que considerar otros factores como la desigualdad, la falta de oportunidades, la caótica urbanización provocada por la explosión demográfica, la falta de acceso a empleos formales, la falta de acceso a la cobertura

15 Miller, Patrick McC., y Plant, Martin, "Drinking, Smoking and Illicit Drug Use among 15 and 16 Year in the United Kingdom”, British Medical Journal, núm. 313, 1998. 
sanitaria mínima, así como la falta de acceso a una educación sólida y de calidad, lo que quizá está en el origen de la delincuencia como factor de exclusión social. Otros factores de tipo psicológico serían la violencia infantil, la violencia familiar, la violencia en los medios de comunicación, así como habilidades poco desarrolladas para la solución de conflictos.

Por tanto, la pobreza no explica por sí sola la violencia juvenil. Habría que buscar la respuesta en la inestabilidad económica, la falta de un modelo sustentable de desarrollo económico, la fragilidad democrática, la ausencia de normas eficaces como consecuencia de la crisis estructural de la familia, la escuela, el Estado, o la Iglesia, de un aparato judicial y policial eficiente, y de fuerzas externas como la drogadicción o el narcotráfico, verdaderos motores de la delincuencia juvenil, estos últimos más bien considerados como consecuencias que como causas de la exclusión social.

\section{LOS JÓVENES Y LA SEGURIDAD SOCIAL. UN ENFOQUE RESPECTO AL FINANCIAMIENTO}

Afrontar los graves problemas de exclusión que sufren los jóvenes respecto a las principales políticas sociales en materia de educación, salud y vivienda está íntimamente relacionado con la falta de acceso a oportunidades laborales y, como consecuencia, a la cobertura de la seguridad social. De acuerdo con cifras del Consejo Nacional de Evaluación para la Política de Desarrollo Social (Coneval), obtenidas de su Encuesta Nacional de Ocupación y Empleo del primer trimestre de 2010,19\% de la población mexicana tiene entre 15 y 24 años. De esta población, 68\% de los jóvenes no cuenta con acceso a la seguridad social, $44.7 \%$ no cuenta con acceso a los servicios de salud y $18.1 \%$ tiene rezago educativo. Además, 3.3 millones de estos jóvenes viven en pobreza extrema, lo que significa que no cuentan con recursos para comprar alimentos de la canasta básica. ${ }^{16}$

La situación en el ámbito internacional en un contexto de profunda recesión económica confirma estas graves carencias. La Organización Internacional del Trabajo (OIT) destaca en su informe Tendencias mundiales del Empleo 2012, que 74.8 millones de jóvenes entre 15 y 24 años estaban desempleados

16 Coneval, Encuesta Nacional de Ocupación y Empleo, primer trimestre de 2010. 
en 2011, ubicando la tasa de desempleo juvenil mundial en $12.7 \% .{ }^{17}$ Destaca que a nivel mundial los jóvenes tienen tres veces más posibilidades que los adultos de estar desempleados.

Por su parte, la OCDE refleja también esta situación al referir que la tasa de desempleo para los jóvenes entre 15 y 24 años de edad fue de $17.4 \%$ en el área de la OCDE, en contraste con el 7\% de los adultos. ${ }^{18}$

En el caso de México, la recesión económica de 2008 ha generado un incremento mayor de la generación de empleo eventual que permanente. De esta forma, en octubre de 2011 la tasa de expansión anual del número de trabajadores asegurados fue de $4.2 \%$, cifra mayor en el caso de trabajadores eventuales $(8.5 \%)$ que en el de permanentes $(3.5 \%)$, situación que ha sido constante en los últimos tres años. ${ }^{19}$

En el caso del empleo informal se aprecia un incremento en México, al pasar de $52.5 \%$ en 2008 a 54.2\% en 2010. La crisis no sólo provocó un aumento del desempleo, sino también una expansión del empleo en el sector informal. Sin embargo, donde es más grave esta situación es en el empleo informal de los jóvenes. De acuerdo con datos de la OIT, el empleo informal total entre los jóvenes de 15 a 24 años en México durante 2010 fue de 65.9\%. ${ }^{20}$

Esta situación ha llevado a diseñar diferentes alternativas en función de crear un mercado laboral más dinámico mediante la remoción de los obstáculos que impiden tanto la participación laboral como la búsqueda del empleo. Algunos de los problemas que enfrentan estas medidas van desde un régimen fiscal poco dado a generar incentivos y estímulos a la contratación de trabajadores jóvenes poco cualificados, hasta un régimen laboral que, pese a regular estrictamente la contratación laboral, enfrenta una débil obligatoriedad, a lo que se acompaña una evasión persistente de contribuciones a la seguridad social bajo el pretexto del incremento en costos.

17 Véase http:// wmw.ilo.org/global/publications/books/global-employment-trends/WCMS_171571 /lang--en/index.htm.

18 Véase http://mww.oecd.org/document/46/0,3746,en_2649_33729_40401454_1_1_1_1,00. btml.

19 OIT, Panorama laboral 2011 América Latina y el Caribe, Oficina Regional para América Latina y el Caribe. Elaboración OIT con base en datos del Instituto Mexicano del Seguro Social (IMSS) sobre la variación interanual de trabajadores cotizantes, enero de 2008-octubre de 2011, p. 37.

20 Ibidem, pp. 65 y ss. 
Ahora bien, las respuestas que se han dado a esta problemática varían en función de las características específicas de cada país. Sin embargo, la mayoría de las intervenciones gubernamentales se enfoca en promover las condiciones necesarias para facilitar la empleabilidad de los jóvenes. Así, una gran parte está destinada a la conclusión de estudios educativos, ${ }^{21}$ a la capacitación laboral ${ }^{22} \mathrm{o}$ a programas para incentivar el primer empleo, ${ }^{23}$ pero no a la promoción de empleos con una cobertura de seguridad social garantizada, o bien a la generación de los incentivos y estímulos económicos necesarios para financiar el acceso a la seguridad social de los jóvenes. En México, por ejemplo, el programa "Primer Empleo" tenía el objetivo de subsidiar durante 12 meses las contribuciones a la seguridad social cuando se reclutaba a personas que nunca habían trabajado, sin importar su edad laboral. Sin embargo, pese a que consideramos que es un primer paso en la dirección correcta, ya está dejando de operar.

\section{CONCLUSIONES}

Aunque la exclusión social se encuentra fuertemente emparentada y superpuesta con la pobreza, se trata de un problema todavía más complejo y con consecuencias tanto o más graves. La exclusión social afecta a la mayoría de la población joven, supone la pérdida de espacios de referencia y pertenencia para las personas en sus procesos de integración social y de construcción de identidad individual y colectiva. El joven excluido, ya sea a consecuencia del desempleo o porque nunca ingresó directamente en las vías adecuadas que ordenan y rigen la vida de las sociedades, deja de ser parte de éstas para transformarse en algo "ajeno".

La exclusión social tiene un amplio referente en la educación. Hemos visto que la educación de los padres y las condiciones sociales juegan un papel fun-

21 En Brasil, el Programa Nacional de Inclusión de Jóvenes (Projovem), o en Estados Unidos el Programa de Becas de Oportunidad para Jóvenes (Youth Opportunity Grants). Véase Informe sobre la seguridad social en América. Niños y seguridad social 2011, México, Conferencia Interamericana de Seguridad Social (CISS) y Centro Interamericano de Estudios de Seguridad Social (CIESS).

22 Como el programa Projoven en Uruguay.

23 Casos de Perú, Colombia y México. 
damental en la exclusión de los jóvenes. Es imposible esperar una sociedad integrada sin una educación y una formación integradoras. La integración no pasa sólo por asegurar un acceso amplio a las oportunidades de educación y formación, sino por incluir a los excluidos en programas y políticas destinados a corregir sus condiciones de desigualdad.

Ahora bien, la construcción de la sociedad del siglo XXI, inmersa en el proceso de globalización, requiere que la modernización tenga como principales protagonistas a los jóvenes, dadas sus potenciales habilidades para la incorporación de las tecnologías de la información y su mayor adaptación a la sociedad del conocimiento. Para ello, las políticas públicas deben enfocarse en un mejor uso de las nuevas tecnologías de la información y la comunicación que faciliten las oportunidades de participación y acceso de los jóvenes en la toma de decisiones que inciden en su vida cotidiana.

Una mayor vinculación entre las instituciones educativas de los niveles medio superior y superior con las necesidades del mercado laboral se hace necesaria para facilitar la capacitación laboral en los jóvenes, de forma que puedan combinarse la educación y el entrenamiento laboral de forma eficiente.

Combatir el empleo informal en la juventud debe convertirse en un objetivo primordial de cualquier política pública orientada a mejorar las condiciones del mercado de trabajo. Las políticas orientadas por estímulos fiscales que promuevan la seguridad social en el empleo juvenil se vuelven un elemento esencial del financiamiento de la seguridad social. No se trata sólo de facilitar el acceso al mercado laboral a los jóvenes, sino de hacerlo en las mejores condiciones posibles. En ese sentido, si bien es importante impulsar el crecimiento del empleo en los jóvenes, ello no debe conducir a descuidar el tratamiento oportuno de los problemas estructurales del mercado laboral como es el caso de un cumplimiento débil de las reglas laborales de contratación laboral, los bajos salarios, o la tolerancia del trabajo informal bajo el pretexto de ser la medida más económica para que los jóvenes obtengan un empleo.

Desde otra perspectiva, se hace indispensable que los jóvenes vayan más allá de los reclamos simbólicos y se enfoquen en la mejora de los sistemas de empleo, el incremento de los ingresos y en recuperar las prestaciones de seguridad social, a manera de compensación en estos tiempos de precariedad y globalización, desempleo y falta de oportunidades. Esto requerirá un cambio en la cultura laboral que debe enfocarse desde las instituciones educativas y 
promoverse por el sector público y la iniciativa privada, con miras a mejorar las condiciones de vida de la sociedad.

Finalmente, y parafraseando a Maffesoli, "lejos del universalismo moderno, el de la Ilustración y el de occidente triunfante, el tribalismo nos recuerda, empíricamente, la importancia del sentimiento de pertenencia, a un lugar, a un grupo, como fundamento esencial de toda vida social...”.

\section{BibliografíA}

BANCO MUNDIAL, El potencial de la juventud: politicas para jóvenes en situación de riesgo en América Latina y el Caribe, Washington, 2007.

BArbería, José Luis, "La clase perdedora”, El País, 7 de abril de 2009.

Bourdieu, Pierre, "La juventud no es más que una palabra", Sociología y Cultura, México, CNCA-Grijalbo, 1990.

CISS, Informe sobre la seguridad social en América. Niños y seguridad social, México, 2011.

Coneval, Encuesta Nacional de Ocupación y Empleo, primer trimestre de 2010.

CURRIE, Elliot, "Crime and Market Society: Lessons from the United States", en Walton, Paul y Young, Jock (eds.), The New Criminology Revisited, Londres, Macmillan, 1998.

Foucault, Michel, Historia de la sexualidad. 1. La voluntad de saber, México, Siglo XXI Editores, 1977.

France, Alan y Wiles, Paul, "Dangerous Futures: Social Exclusion and Routh Work in Late Modernity", Jones-FINER, Catherine y NeLLIS, Mike (eds.), Crime and Social Exclusion, Oxford, Blackwell, 1998.

GidDENS, Anthony, Sociología, Madrid, Alianza, 2001.

HOPENHAYN, Martin, "Inclusión social y exclusión social en la juventud latinoamericana", Pensamiento iberoamericano, España, AECID y Fundación Carolina, núm. 3, 2008.

MAFFESOLI, Michel, El tiempo de las tribus. El ocaso del individualismo en las sociedades posmodernas, México, Siglo XXI Editores, 2004.

Medina Carrasco, Gabriel, Aproximaciones a la diversidad juvenil, México, El Colegio de México, 2000. 
Miller, Patrick McC. y Plant, Martin, "Drinking, Smoking and Illicit Drug Use among 15 and 16 Years in the United Kingdom", British Medical Journal, núm. 313, 1996.

RodrígueZ, Ernesto, "Políticas públicas de la juventud en América Latina: Experiencias”, Pensamiento iberoamericano, España, AECID y Fundación Carolina, núm. 3, 2008.

Reguillo, Rossana, "Las múltiples fronteras de la violencia: jóvenes latinoamericanos entre la precarización y el desencanto", Pensamiento iberoamericano, núm. 3, España, AECID, 2008.

YounG, Jock, "Breaking Windows: Situating the New Criminology", en Walton, Paul y Young, Jock (eds.), The Exclusive Society: Social Exclusion. Crime and Difference in Late Modernity, Londres, Sage, 1998. Ty 